\title{
STRUCTURAL ANNOTATION OF EM IMAGES BY GRAPH CUT
}

\author{
Hang Chang, Manfred Auer, and Bahram Parvin \\ Life Sciences Division \\ Lawrence Berkeley National Laboratory, Berkeley, CA 94720 \\ hchang@lbl.gov
}

\begin{abstract}
Biological images have the potential to reveal complex signatures that may not be amenable to morphological modeling in terms of shape, location, texture, and color. An effective analytical method is to characterize the composition of a specimen based on user-defined patterns of texture and contrast formation. However, such a simple requirement demands an improved model for stability and robustness. Here, an interactive computational model is introduced for learning patterns of interest by example. The learned patterns bound an active contour model in which the traditional gradient descent optimization is replaced by the more efficient optimization of the graph cut methods. First, the energy function is defined according to the curve evolution. Next, a graph is constructed with weighted edges on the energy function and is optimized with the graph cut algorithm. As a result, the method combines the advantages of the level set method and graph cut algorithm, i.e., "topological" invariance and computational efficiency. The technique is extended to the multiphase segmentation problem; the method is validated on synthetic images and then applied to specimens imaged by transmission electron microscopy(TEM).
\end{abstract}

Index Terms - Interactive learning, Active Contour, Graph Cut, Texture, Segmentation, Electron Microscopy

\section{INTRODUCTION}

Biological specimens have a complex phenotypic signature requiring multiscale representations of their information content. At the coarse scale, we are interested in the composition of various tissue compartments, which can be interactively specified in a few small areas. These candidate patterns can then be identified in a mosaic of images for compositional analysis. As a result, the methods enables the quantification and comparative analysis of tissue sections under different experimental conditions. The proposed method couples active contour models $[1,2]$ with the graph cut optimization method based on the link established by [3] to demonstrate superior

RESEARCH WAS PARTIALLY SUPPORTED BY A GRANT NUMBER 007G18 FROM ENERGY BIOSCIENCES INSTITUTE UNDER CONTRACT NUMBER DE-AC02-05CH1131 WITH THE UNIVERSITY OF CALIFORNIA. performance, which includes "topologically" free curve evolution, limited leakage, and an efficient optimization of the objective energy function. The method is applied to the segmentation and compositional analysis of specimens that have been imaged by TEM. As a result, structural features corresponding to organelles and various molecular complexes can be identified. Unlike fluorescence microscopy, patterns of image formation through electron microscopy are complex; minute spatial features of limited perceptual significance may be important; and texture and contrast varies widely from one complex to another. Therefore, we have opted for a trainable system that elicits important spatial features of interest. Figure 1 shows the work flow of our approach.



Fig. 1. Work flow of our approach.

This rest of this paper consists of the following sections: Section 2 provides a summary of the active contour models without edges and a description of the graph cut method; Section 3 outlines the details of our approach; Section 4 compares our method with the traditional level set approach and demonstrates its effectiveness; and Section 5 concludes the paper.

\section{RELATED WORK}

In this section, we introduce Chan and Vese's multiphase active contour model and the principles of graph cut approach.

\subsection{Active Contour Model with Multiple Phases}

Chan and Vese generalized their system [1] into the multiphase(multi-class) level set model in [2]. Their implementation is quite different from other methods, which assign a level set function to each phase. Their framework reduces the number of level set functions from $M$ (number of phases) to $\log _{2} M$ by introducing the "vector level set function" $\Phi=$ $\left(\phi_{1}, \ldots, \phi_{m}\right) \mid \phi_{i}: \Omega \rightarrow \mathbb{R}$, and the "vector Heaviside function"

$H(\Phi)=\left(H\left(\phi_{1}\right), \ldots, H\left(\phi_{m}\right)\right) \mid H\left(\phi_{i}\right) \in\{0,1\}, 1 \leq i \leq m=\log _{2} M$ 
For each phase $i, c_{i}$ is defined to be the mean intensity in region $i$, and $\mathbb{X}_{i}$ is defined to be the characteristic function of region $i$. As a result, the objective energy function could be written as:

$$
F_{M}=\mu \frac{1}{2} \sum_{i=1}^{M=2^{m}} \int_{\Omega}\left|\nabla \mathbb{X}_{i}\right|+\sum_{i=1}^{M=2^{m}} \int_{\Omega}\left(u_{0}(x, y)-c_{i}\right)^{2} \mathbb{X}_{i} d x d y
$$

where $u_{0}(x, y)$ is the original image. The model is further simplified by replacing the length term by $\sum_{i=1}^{m} \int_{\Omega}\left|\nabla H\left(\phi_{i}\right)\right|$, which provides satisfactory results [2], although it leads to the redundant counting of a curve segment subset. The final energy for minimization is formulated as

$$
F_{M}=\mu \sum_{i=1}^{m} \int_{\Omega}\left|\nabla H\left(\phi_{i}\right)\right|+\sum_{i=1}^{M=2^{m}} \int_{\Omega}\left(u_{0}(x, y)-c_{i}\right)^{2} \mathbb{X}_{i} d x d y
$$

The optimization problem is then solved by the gradient descent method based on the corresponding Euler-Lagrange equations.

\subsection{Graph Cut Method}

In the context of segmentation, this method represents the image as a graph $G=\langle\bar{V}, \bar{E}\rangle$, where $\bar{V}$ and $\bar{E}$ correspond to the sets of nodes and edges, respectively. Usually, nodes and edges are pixels, and their relationship, which is typically a 4- or 8-connected configuration. Additionally, there are some special nodes called terminals in the graph structure, which correspond to a set of labels $(\mathcal{L})$. The segmentation problem is to assign a unique label $x_{p} \in \mathcal{L}$ to each node $p \in \mathcal{P}$, and the image cutout is performed by minimizing the Gibbs energy $E(X)[4]$ :

$$
E=\sum_{p \in \mathcal{P}} E_{1}\left(x_{p}\right)+\sum_{(p, q) \in \mathbb{N}, x_{p} \neq x_{q}} E_{2}\left(x_{p}, x_{q}\right)
$$

where $\mathbb{N}$ is the neighborhood system; $E_{1}\left(x_{p}\right)$ is the likelihood energy encoding the fitness cost for assigning $x_{p}$ to $p$; and $E_{2}\left(x_{p}, x_{q}\right)$ is the smoothness energy denoting the cost when the labels of adjacent nodes $p$ and $q$ are $x_{p}$ and $x_{q}$, respectively. Based on the number of terminals, the graph cut could be classified into two groups: classical two-label graph cut and multilabel graph cut. For the two-label labeling problem, polynomial-bound methods exist for optimizing directed weighted graphs with two terminals. In the case of multilabel graphs, Boykov [5] proposed two effective optimization algorithms, known as $\alpha$ - $\beta$-swap and $\alpha$-expansion.

\section{APPROACH}

Suppose that we want to divide an image into $M=|\mathcal{L}|$ parts. Each part is described as $\Omega_{i}, 1 \leq i \leq M$, so that

$$
\begin{array}{r}
\Omega_{i} \cap \Omega_{j}=\emptyset, i \neq j \\
\Omega_{1} \cup \Omega_{2} \cdots \cup \Omega_{M}=\Omega
\end{array}
$$

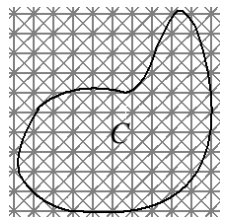

(a)



(b)
Fig. 2. (a) Eight-neighborhood $2 \mathrm{D}$ grid. (b) One family of lines.

Let (i) $u^{k}(p)$ be the $k^{t h}$ feature of pixel $p$, and (ii) $\mathbf{p}_{i}^{k}$ be the probability function of the $k^{t h}$ feature of region $i, 1 \leq i \leq M$. The objective energy function to be minimized is formulated as:

$E=\mu \cdot$ BoundaryLength $-\sum_{i=1}^{M} \sum_{k=1}^{N} \lambda_{i}^{k} \int_{\Omega_{i}} \log \mathbf{p}_{i}^{k}\left(u^{k}(p)\right) d p$

in which $\mu$ and $\lambda_{i}^{k}$ are constant weights. The first term ensures the smoothness of the segmented region boundary, and the last term ensures the similarity within each segmented region in the feature space. Within the multiphase level set approach, as described in Section 2.1, the objective energy function (4) can be written as:

$E=\mu \sum_{i=1}^{m} \int_{\Omega}\left|\nabla H\left(\phi_{i}\right)\right|-\sum_{i=1}^{M=2^{m}} \sum_{k=1}^{N} \lambda_{i}^{k} \int_{\Omega} \log \mathbf{p}_{i}^{k}\left(u^{k}(p)\right) \mathbb{X}_{i} d p$

Although this function can be optimized by using the gradient descent method, we have chosen the multilabel graph cut method to perform the energy minimization for better efficiency. Let $\alpha_{i}=i, 1 \leq i \leq M$ be the node label, and $x_{p} \in\left\{\alpha_{1}, \ldots, \alpha_{M}\right\}$. In a discrete graph, the energy functional (4) could be written as:

$$
\begin{aligned}
E & =\mu \cdot \text { BoundaryLength } \\
& -\sum_{i=1}^{M} \sum_{k=1}^{N} \lambda_{i}^{k} \sum_{\Omega} \log \mathbf{p}_{i}^{k}\left(u^{k}(p)\right) \delta_{\alpha_{i}}\left(x_{p}\right)
\end{aligned}
$$

In order to optimize the active contour model via graph cut, the Euclidean length, represented as $|C|_{\epsilon}$, needs to be approximated by specifically designed n-links (edges between neighboring pixels) of the graph model. For eight-neighborhood system illustrated in Figure 2, it has been shown( by Function [3] ) that this distance is given by:

$$
|C|_{\epsilon} \approx \sum_{k} n_{c}(k) \cdot \frac{\delta^{2} \cdot \triangle \phi_{k}}{2 \cdot\left|e_{k}\right|}
$$

where $n_{c}(k)$ is the number of intersections of the curve $C$ with the $k^{t h}$ family of edge lines; $\delta$ is the cell-size of the grid; $\left|e_{k}\right|$ is the Euclidean length of vector $e_{k}$; and $\triangle \phi_{k}$ is the angular difference between the $k^{t h}$ and $(k+1)^{t h}$ edge lines, 
$\triangle \phi_{k}=\phi_{k+1}-\phi_{k}$. It has been shown that with constant edge weights of

$$
w_{k}=\frac{\delta^{2} \cdot \triangle \phi_{k}}{2 \cdot\left|e_{k}\right|}
$$

for each family of edge lines, the Euclidean length of $C$ can be approximated by the cut length, which is defined as the sum of weights of broken n-links in the graph grid. In our implementation, we chose to use the eight-neighborhood system, and the structure of graph $G=\langle\bar{V}, \bar{E}\rangle$ is as follows: $\bar{V}$ contains all the pixels, $\mathcal{P}$, in the input image and $M$ terminals. Each $p \in \mathcal{P}$ is connected to every terminal with edge $t_{p}^{i}$, $1 \leq i \leq M$. Each pair of $(p, q)$ in the neighborhood system $\mathbb{N}$ is connected with edge $w_{\{p, q\}}$. The edge weights of the graph, which encode the energy terms of the active contour model, are expressed in Table 1 . Then, we apply the $\alpha-\beta$ -

\begin{tabular}{|c|c|c|}
\hline Edge & Weight & For \\
\hline \hline$t_{p}^{i}$ & $-\sum_{k} \lambda^{k} \log \mathbf{p}_{i}^{k}\left(u^{k}(p)\right)$ & $p \in \mathcal{P}, 1 \leq i \leq M$ \\
\hline$w_{\{p, q\}}$ & $\frac{\pi}{8 T}$ & $\{p, q\} \in \mathbb{N}, T \in\{1, \sqrt{2}\}$ \\
\hline
\end{tabular}

Table 1. Edge weights for the multilabel graph construction based on the eight-neighborhood system.

swap algorithm to optimize the estimation, and the algorithm flow is shown as follows.

$$
\alpha-\beta \text {-swap }
$$

1. Start with an arbitrary labeling $X$

2. Set success $=0$

3. For each pair of labels $\{\alpha, \beta\} \subset \mathcal{L}$ :

(a) Find $\hat{X}=\min E\left(X^{\prime}\right)$ among $X^{\prime}$ within one $\alpha-\beta$ swap of $X$

(b) If $E(\hat{X})<E(X)$, set $X=\hat{X}$ and success $=1$

4. If success $=1$, go to 2

\section{Return $X$}

In each cycle (steps 2-4), the algorithm performs an iteration (step 3) for each pair of labels, and stops when there is no improvement. Each cycle takes $|\mathcal{L}|^{2}$ iterations. It has been shown that (i) this method terminates in $O(|\mathcal{P}|)$ cycles, and (ii) the final energy under labeling $X$ is a local minimum with respect to a swap [6], where the optimal swap move in Step 3(a) is computed by graph cuts. For the labeling result $X$ and the pair of chosen labels $(\alpha, \beta)$, the graph is represented as $G_{\alpha \beta}=\left\langle\bar{V}_{\alpha \beta}, \bar{E}_{\alpha \beta}\right\rangle$, where $\bar{V}_{\alpha \beta}$ contains two terminals $\alpha, \beta$; all pixels in the set $\mathcal{P}_{\alpha \beta}=\mathcal{P}_{\alpha} \cup \mathcal{P}_{\beta}$; and each pixel $p \in \mathcal{P}_{\alpha \beta}$ is connected to the terminals $\alpha$ and $\beta$ by edges $t_{p}^{\alpha}$ and $t_{p}^{\beta}$. Each pair of pixels $\{p, q\} \in \mathbb{N}$ is connected by an edge $w_{\{p, q\}}$. The weights of the edges are shown in Table 2. Furthermore, the optimal $\alpha-\beta$ swap from $X$ is $\hat{X}=X^{\mathcal{C}}$, where $C$ is the minimum cut on $G_{\alpha \beta}$, and $X^{\mathcal{C}}$ is the labeling that corresponds to cut $\mathcal{C}[5]$.

\begin{tabular}{|c|c|c|}
\hline Edge & Weight & For \\
\hline \hline$p \rightarrow \alpha$ & $E_{1}\left(x_{p}=\alpha\right)+$ & $p \in \mathcal{P}_{\alpha \beta}$ \\
& $\sum_{q \in \mathbb{N}_{p}, q \notin \mathcal{P}_{\alpha \beta}} E_{2}\left(x_{p}=\alpha, x_{q}\right)$ & \\
\hline$p \rightarrow \beta$ & $E_{1}\left(x_{p}=\beta\right)+$ & $p \in \mathcal{P}_{\alpha \beta}$ \\
& $\sum_{q \in \mathbb{N}_{p}, q \notin \mathcal{P}_{\alpha \beta}} E_{2}\left(x_{p}=\beta, x_{q}\right)$ & \\
\hline & $E_{2}(\alpha, \beta)$ & $\{p, q\} \in \mathbb{N}$ \\
$w_{\{p, q\}}$ & & $p, q \in \mathcal{P}_{\alpha \beta}$ \\
\hline
\end{tabular}

Table 2. Edge weights of graph $G_{\alpha \beta}$ for $\alpha$ - $\beta$ swap.

\section{EXPERIMENTAL RESULTS}

To validate our method, we have tested our implementation on synthetic and scientific images. The texture features are extracted by a bank of gabor filters. The method can also incorporate color data or hyperspectral data as additional feature values. The input image together with the user-specified stroke provides the seeds for the corresponding feature-based representation of each region. The Gaussian mixture model (GMM, with 10 components) provides a model for color and texture distributions based on user-provided information (details can be found in [7]). Let (i) $G M M_{i}^{C}$ and $G M M_{i}^{T}$ represent the texture model for the $i^{\text {th }}$ region, respectively, where (i) $1 \leq i \leq M$, and (ii) $\mathbf{p}_{i}^{C}(p)$ and $\mathbf{p}_{i}^{T}(p)$ refer to the probabilities of contrast and texture features of pixel $p$ belonging to the $i^{t h}$ region. Then

$$
\mathbf{p}_{i}^{C}(p)=\frac{G M M_{i}^{C}(p)}{\sum_{j=1}^{M} G M M_{j}^{C}(p)}, \mathbf{p}_{i}^{T}(p)=\frac{G M M_{i}^{T}(p)}{\sum_{j=1}^{M} G M M_{j}^{T}(p)}
$$

The graph is then constructed according to Table 1 and optimized with the $\alpha$ - $\beta$-swap algorithm. Figure 3 shows the labeling result on an synthetic image and compares the result with the traditional level set method. It is clear that the proposed method reduces fragmentation, and the computational complexity of our method is comparable to the traditional level set method. This is because the traditional level set method is iterative, while the graph cut method is not. Figure 4 indicates the results of our method on samples that are imaged by TEM. Note that the images are generally noisy, and different components of the micro-anatomy have unique textures. Although it is beyond the scope of this paper to review previous research on the segmentation of samples imaged by electron microscopy, we must add that the fast marching method initiating from interest point operators has been proposed [8]. We 
suggest that the proposed region-based method (as opposed to the gradient-based fast marching method) be coupled with the prior texture model to provide a more robust solution to the segmentation problem.



(a)



(b)



(c)

Fig. 3. (a) Original image with user input samples. (b) Segmentation result from the traditional level set method $(t=3.3 \mathrm{sec})$. (c) Segmentation result from our model $(t=$ $2.7 \mathrm{sec})$.

\section{CONCLUSION}

In this paper, we proposed a system for the compositional analysis of complex patterns that are imaged through electron microscopy, which is an extension of our previous work [9]. We suggest that, segmentation and subsequent compositional analysis can be enabled for larger size-images by providing examplar patterns. Our proposed method couples the new active contour model of Chan and Vese's model with the graph cut algorithm. The level set formulation incorporates multiphase implementation for global differentiation of the scenes into distinct regions. Our method has the advantages of "topological" freedom, minimal leakage, and high efficiency in energy minimization. These advantages are ensured by the intrinsic properties of the level set method and graph cut algorithm. The behavior of the method has been validated on synthetic images, and then applied to a specimen imaged by TEM for population analysis. The potential limitations of our method are twofold: (i) underlying feature properties are represented with Gaussian distributions, and (ii) the absence of higher-level constraints for eliminating false positive regions. In the first case, we plan to use generative models to achieve a more parameter-free representation of the underlying image features. In the second case, we plan to couple low-level image features with higher-level constraints such as shape [10] and other contextual features.

\section{REFERENCES}

[1] T. F. Chan, L. A. Vese, Active contours without edges, IEEE Transactions on Image Processing 10 (2001) 266-277.


(b)


(c)


Fig. 4. Segmentation results for samples imaged through transmission electron microscopy with the trained regions (left column) and automated labeling (right column): (a) 70nm thick section through a zebra fish notochord, (b) multi-phase results of a human mammary epithelial cell, and (c) a plant cell and its compartments.

[2] L. Vese, T. Chan, A multiphase level set framework for image segmentation using the mumford and shah model, International Journal of Computer Vision 50 (3) (2002) 271-293.

[3] Y. Boykov, V. Kolmogorov, Computing geodesics and minimal surfaces via graph cuts, in: Proc. of IEEE ICCV, 2003.

[4] S.Geman, D.Geman, Stochastic relaxation, gibbs distribution and the bayesian restoration of images, In IEEE Transaction on PAMI 6 (6) (1984) 721-741.

[5] Y.Boykov, O. Veksler, R. Zabih, Fast Approximate Energy Minimization via Graph Cuts, In IEEE Transaction on PAMI 23 (11) (2001) 1222-1239.

[6] O. Veksler, Efficient graph-based energy minimization methods in computer vision., $\mathrm{PhD}$ thesis, Cornell University.

[7] H. Chang, R. Defilippis, T. Tlsty, B. Parvin, Graphical methods for quantifying macromolecules through bright field imaging, Bioinformatics 25 (8) (2008) 1070-1075.

[8] C. Bajaj, Z. Yu, M. Auer, Volumetric feature extraction and visualization of tomographic molecular imaging, Journal of Structural Biology 144 (2003) 132-143.

[9] H. Chang, Q. Yang, M. Auer, B. Parvin, Modeling of front evolution with graph cut optimization, in: Proceedings of the IEEE ICIP, 2007, pp. 241-244.

[10] H. Chang, Q. Yang, B. Parvin, A bayesian approach for image segmentation with shape priors, in: Proceedings of the IEEE CVPR, 2008, pp. 1-8. 